\title{
Prevalencia de malnutrición en menores de 5 años. Comparación entre parámetros OMS y su adaptación a Colombia
}

\author{
Prevalence of malnutrition in children under 5 years of age. Comparison between OMS parameters and their adaptation \\ to Colombia
}

Nathaly Barrera Dussán 1* orcid.org/0000-0002-4310-7380

Jorge Andrés Ramos-Castañeda² orcid.org/0000-0003-3297-0012

1 Programa de Enfermería, Universidad Surcolombiana, Neiva, Colombia

2 Grupo de Epidemiología y Salud Pública, Universidad Surcolombiana, Neiva, Colombia

Fecha de recepción: Mayo 23-2019 Fecha de revisión: Septiembre 11 - 2019 Fecha de aceptación: Diciembre 19 - 2019

Barrera Dussán N, Ramos-Castañeda JA. Prevalencia de malnutrición en menores de 5 años. Comparación entre parámetros OMS y su adaptación a Colombia. Univ. Salud. 2020;22(1):91-95. DOI: https://doi.org/10.22267/rus.202201.179

\section{Resumen}

Introducción: La Organización Mundial de la Salud (OMS) establece los patrones de crecimiento de niños menores de 5 años. Colombia adoptó indicadores antropométricos y puntos de corte para clasificar el estado nutricional. Sin embargo, para desnutrición se considera Peso/Talla en vez del Índice de Masa Corporal (IMC). Objetivo: Identificar la prevalencia de malnutrición en menores de 5 años del área urbana del municipio de Palermo, Huila (Colombia), comparando los resultados con los patrones de la OMS y resolución 2465 de 2016 en el parámetro IMC/Edad. Materiales y métodos: Estudio de corte transversal, con enfoque analítico con 254 niños menores de 5 años del municipio de Palermo. Resultados: Según IMC/edad el 11,81\% de los menores presentaron algún tipo de malnutrición: $1,9 \%$ desnutrición y 9,8\% sobrepeso y obesidad. Un 25,5\% estuvieron en riesgo de presentarlo, siendo mayor el sobrepeso $(17,7 \%)$. Según parámetros OMS el 12,2\% presentaron malnutrición: 9,8\% por exceso y 2,4\% por déficit. El $18,5 \%$ y $7,9 \%$ tuvieron riesgo de sobrepeso y bajo peso respectivamente. Conclusión: No existen diferencias al comparar la clasificación nutricional entre la Resolución y los parámetros de la OMS.

Palabras clave: Desnutrición; sobrepeso; obesidad; cuidadores; determinantes sociales de la salud (Fuente: DeCS, Bireme).

\begin{abstract}
Introduction: The World Health Organization (WHO), has established the growth patterns of children under 5 years of age. Colombia has adopted anthropometric indicators and cut-off points to classify nutritional status. However, weight/height are the only measurements taken into account for defining malnutrition, instead of Body Mass Index (BMI). Objective: To identify malnutrition prevalence in children who are younger than 5 years of age and live in the metropolitan area of the municipality of Palermo-Huila (Colombia), comparing the results with the WHO reference standards and with the IMC/Age parameter of Resolution 2465 of 2106. Materials and methods: A cross-sectional study with an analytical approach was conducted on 254 children younger than 5 years old from Palermo. Results: According to the BMI/Age parameter $11.81 \%$ of the children showed some nutrition problem: $1.9 \%$ malnutrition and $9.8 \%$ overweight and obesity. $25.5 \%$ of children were at risk of having some type of malnutrition, with the risk of overweight (17.7\%) being the highest. According to WHO parameters, $12.2 \%$ of child participants showed malnutrition: $9.8 \%$ due to excess and $2.4 \%$ because of a deficient intake of nutrients. It was observed that $18,5 \%$ and $7,9 \%$ of the minors were at risk of being overweight and underweight, respectively. Conclusions: There are no differences when comparing the nutritional classification of the Resolution with the one based on the WHO parameters.
\end{abstract}

Keywords: Undernutrition; overweight; obesity; caregivers; social determinants of health (Source: DeCS, Bireme).

*Autor de correspondencia

Nathaly Barrera Dussán

e-mail: natybadu@gmail.com 


\section{Introducción}

El estado nutricional en condiciones normales es el resultante del balance entre lo consumido y lo requerido, lo cual está determinado por la calidad y cantidad de nutrientes de la dieta y por su utilización completa en el organismo(1).

De acuerdo a la Organización Mundial de la Salud (OMS), la malnutrición abarca la desnutrición, el sobrepeso, la obesidad y el desequilibrio de micronutrientes, que se genera por un desbalance entre la ingesta de nutrientes y las necesidades nutricionales. Para el año 2016, se calcula que aproximadamente 155 millones de niños presentaron retraso del crecimiento, mientras que 41 millones tenían sobrepeso u obesidad(2).

En Colombia la malnutrición continúa siendo un problema de salud pública que afecta a los menores de cinco años, generando consecuencias a corto y largo plazo para el desarrollo físico y cognitivo, y que determinan el aumento en la oportunidad de aparición de complicaciones cardiovasculares en la edad adulta; consecuencias que a su vez pueden alterar la productividad de una región ${ }^{(3-5) .}$

El seguimiento realizado por la Secretaría de Salud Departamental del Huila a los indicadores nutricionales en población menor de 5 años del municipio de Palermo, reporta: 6,5\% de recién nacidos con bajo peso al nacer, $1,85 \%$ de la población en estado de desnutrición aguda, 5,99\% desnutrición crónica, 1,18\% desnutrición global, 4,51\% sobrepeso y el $0,89 \%$ en condición de obesidad(6). En Palermo el $6,7 \%$ y 9,8\% de los menores de 5 años presentan malnutrición por déficit o por exceso, respectivamente. Según estudios realizados en el municipio, existe asociación entre el estado nutricional y los determinantes sociales de la salud(7).

Desde 1970 se utilizaban las tablas de referencia de crecimiento del National Center for Health Statistic/OMS para la evaluación de crecimiento en los niños, sin embargo, los resultados no permitían su uso adecuado a nivel internacional teniendo en cuenta que la tabla de referencia se basaba en datos de una muestra limitada de niños de Estados Unidos(8). En el 2006 la OMS publicó un nuevo patrón basado en el niño alimentado con leche materna, con una muestra representativa, permitiendo mejorar la precisión en la evaluación de los resultados. Estos patrones muestran cómo debería ser el crecimiento de los niños y niñas menores de cinco años, cuando sus necesidades de alimentación y cuidados de salud son satisfechos, en cualquier parte del mundo(9).

Colombia a través de la Resolución 2465 de 2016, adoptó los indicadores antropométricos, patrones de referencia y puntos de corte para realizar la clasificación antropométrica del estado nutricional conforme con los patrones de crecimiento publicados en los años 2006 por la OMS. Sin embargo, la Resolución según el indicador IMC, no tiene en cuenta la clasificación para desnutrición como si lo hace la OMS, sino que lo hace con base al indicador peso para la talla, utilizando el término desnutrición aguda. Estas diferencias podrían en algún momento generar cambios en los datos estadísticos en estudios que influyen directamente en la toma de decisiones para los programas de salud pública infantil(10).

Teniendo en cuenta la diferencia en el parámetro IMC/edad entre la Resolución 2465 de 2016 y los parámetros de medición de la OMS en el mismo indicador, en el presente artículo se presenta la comparación de la prevalencia de malnutrición en menores de 5 años al caracterizar los resultados según ambos parámetros.

\section{Materiales y métodos}

\section{Diseño}

Se realizó un estudio descriptivo de corte transversal con enfoque analítico, que permitió determinar la prevalencia de malnutrición en los niños y niñas menores de 5 años caracterizados en la base del SISBEN (Sistema de Identificación de Potenciales Beneficiarios de Programas Sociales) del área urbana del municipio de Palermo, y comparar los hallazgos teniendo en cuenta los parámetros de la Resolución 2465 de 2016 y los de la OMS.

La descripción del municipio de Palermo donde se llevó a cabo la investigación, la información de la muestra y los detalles para la recolección de la información, fueron descritas en un artículo recientemente publicado ${ }^{(7)}$.

\section{Análisis estadístico}

Para determinar la prevalencia de malnutrición se calculó la puntuación $z$ a través del software recomendado por la OMS, ANTHRO para menores de 5 años, versión 3.2.2 disponible para descarga gratuita en la página web de dicha organización. Se determinaron los puntos de corte para: peso para la 
edad, peso para la talla, talla para la edad e Índice de Masa Corporal (IMC) para la edad, tomando como criterios para la clasificación de cada tipo de puntuación, la determinada por la Resolución 2465 de 2016(10). Posteriormente, se determinó la clasificación nutricional según patrones de referencia de la OMS para IMC/edad(11) y se compararon los resultados. Se realizó la estimación con intervalos de confianza del 95\% con apoyo del software Epidat 4.2, utilizando el tamaño de la muestra seleccionada y el número de menores en cada clasificación.

\section{Consideraciones éticas}

Según el artículo 11 de la Resolución 8430 de 1993(12) el estudio se clasificó como una investigación de riesgo mínimo. Se solicitó el consentimiento informado de los padres de los menores de 5 años. La investigación fue aprobada por el Comité de Ética de la Universidad Surcolombiana, por el acta número 10 del 15 de Noviembre de 2016.

\section{Resultados}

Teniendo en cuenta la Resolución 2465 de 2016 el $11,81 \%$, de los menores presentaron algún tipo de malnutrición (desnutrición aguda moderada, desnutrición aguda severa; sobrepeso u obesidad), siendo 1,9\% desnutrición y 9,8\% sobrepeso y obesidad. Un $25,5 \%$ de los menores están en riesgo de presentar algún tipo malnutrición, siendo el riesgo de sobrepeso $(17,7 \%)$ el porcentaje más alto en la población estudiada. Al comparar estos resultados con los indicadores IMC/edad según parámetros OMS se obtienen los siguientes resultados: el 12,2\% presentaron malnutrición: 9,8\% por exceso y $2,4 \%$ por déficit. Se observó $18,5 \%$ de los menores con riesgo de sobrepeso y un 7,9\% con riesgo de bajo peso (Tabla 1).

Al asociar exceso de peso, con algunos de los determinantes sociales, no se encontró asociación estadísticamente significativa para este tipo de malnutrición, con ninguno de los dos patrones de referencia. Para la variable desnutrición aguda (que incluye desnutrición aguda, moderada y severa según Resolución 2465), se encontró asociación estadísticamente significativa con disposición inadecuada de basuras ( $p=0,03$; $\mathrm{RP}=6,083$; IC: $95 \%$ 4,2 - 8,7). Para la variable desnutrición, que incluye desnutrición y desnutrición severa según OMS, no se encontró asociación estadísticamente significativa con ningún determinante social.

Según parámetros de la Resolución 2465 de 2016 al realizar el análisis de regresión logística, se encontró probabilidad de aumento en el riesgo de aparición de desnutrición crónica, explicada en un 5\%; al asociarla con la presencia de determinantes sociales: hacinamiento y bajo ingreso económico, encontrando significancia en el modelo $(p=0,0057)$. Ésta última variable incrementa la probabilidad de aparición de desnutrición crónica 0,17 veces. $(p=0,02)$. Para la desnutrición aguda no se encontró un modelo estadísticamente significativo (Tabla 2).

Utilizando los parámetros de la OMS, se encontró probabilidad de aumento en el riesgo de aparición de desnutrición, explicada en un 2\%; al asociarla con la presencia de determinantes sociales: hacinamiento, disposición inadecuada de basuras y bajo ingreso económico encontrando significancia en el modelo $(p=0,04)$.

Tabla 1. Comparación prevalencias de clasificación nutricional según parámetros Resolución 2465 y OMS

\begin{tabular}{|c|c|c|c|c|}
\hline Clasificación nutricional & $\begin{array}{l}\text { Prevalencia según } \\
\text { parámetro } 2465(\%)\end{array}$ & $\begin{array}{l}\text { Intervalos de } \\
\text { confianza }\end{array}$ & $\begin{array}{l}\text { Prevalencia según } \\
\text { parámetro OMS (\%) }\end{array}$ & $\begin{array}{c}\text { Intervalos de } \\
\text { confianza }\end{array}$ \\
\hline Peso normal & 62.6 & $57.6-68.8$ & 61.42 & $58.1-67.3$ \\
\hline Desnutrición & 1,97 & $0,64-4,53$ & 2,36 & $0,87-5,07$ \\
\hline Sobrepeso & 6,29 & $3,04-10,03$ & 5,90 & $3,34-9,55$ \\
\hline Obesidad & 3,54 & $1,63-6,62$ & 3,94 & $1,9-7,12$ \\
\hline Riesgo de bajo peso & 17,72 & $13,23-22,9$ & 18,50 & $13,92-23,83$ \\
\hline Riesgo de sobrepeso & 7,87 & $4,88-11,9$ & 7,87 & 4,88-11,9 \\
\hline
\end{tabular}


Tabla 2. Regresión logística DNT Crónica y determinantes: hacinamiento y bajo ingreso económico en menores de 5 años

\begin{tabular}{lcccc}
\hline \multicolumn{1}{c}{ Variables } & $\boldsymbol{\beta}$ & $\boldsymbol{p}$ & OR & $\begin{array}{c}\text { Intervalos de } \\
\text { confianza }\end{array}$ \\
\hline Hacinamiento & 0,162 & 0,143 & 2,00 & $0,74-5,42$ \\
Bajo ingreso & 0,170 & 0,019 & 2,31 & $1,14-4,67$ \\
económico & & & & \\
\hline
\end{tabular}

\section{Discusión}

En Argentina, Sguassero et al., realizaron un estudio transversal para analizar los resultados antropométricos según las referencias nacionales e internacionales, comparando las prevalencias entre los parámetros de la OMS y la NCHS (National Center for Health Statistics). Se encontró una prevalencia de bajo peso 1,7 veces mayor en los menores de 6 meses al utilizar los estándares de la OMS comparado con el estándar nacional(13). Al comparar este resultado con el presente estudio la prevalencia de desnutrición también aumentó pero la diferencia fue de sólo 1 caso. En el estudio de Argentina la prevalencia de sobrepeso también aumentó con los estándares de la OMS, siendo en este parámetro contrario a estos resultados donde disminuyó en 1 caso.

En Colombia se han realizado estudios de comparación entre estándares OMS 2006-2007 y la NCHS/CDC 2000, como es el caso del estudio realizado por Sepúlveda et al., quienes compararon la clasificación nutricional de niños entre los 2 y 18 años de edad, encontrando que para el indicador IMC/edad la prevalencia de bajo peso fue un $1 \%$ más con parámetros de la OMS y el sobrepeso fue del 14\% según parámetros OMS y $8 \%$ por CDC. Para la clasificación de obesidad la diferencia fue del $1 \%$ siendo mayor con los entándares de la OMS(14). Para este estudio los resultados no mostraron grandes diferencias, sin embargo coincide el incremento tanto en desnutrición como con obesidad según parámetros OMS.

Alvarez et al., realizaron en Medellín (Colombia) un estudio comparativo entre estándares de la OMS y su adaptación para colombia propuesta a través de la Resolución 2121 de 2010, donde lograron observar menores prevalencias tanto en el indicador peso/talla e IMC/edad de peso adecuado y mayor sobrepeso y obesidad en menores de 5 años usando la clasificación colombiana en relación con la descrita por la OMS(15).
En el presente estudio, al comparar los resultados de los parámetros entre OMS y Resolución 2465 de 2016, no se encuentran grandes diferencias, coexisten ambos extremos de malnutrición a nivel del municipio, tanto por déficit como por exceso. Es importante mencionar que la Resolución aclara que en menores de cinco años el Peso para la Edad (P/E) y el IMC para la Edad (IMC/E) se utiliza únicamente para análisis poblacionales. Mientras que los demás indicadores se utilizan tanto para análisis poblacionales como individuales(10).

\section{Conclusiones}

No existen grandes diferencias al comprar la clasificación nutricional entre la Resolución 2465 y los parámetros de la OMS.

Conflicto de intereses: Los autores declaramos no tener conflictos de interés.

\section{Referencias}

1. Ministerio de salud y protección social. Prevención de la malnutrición o desnutrición. Guía técnica: buenas prácticas para la seguridad del paciente en la atención en salud. Bogotá. 2010.

2. Organización Mundial de la Salud. Malnutrición: datos y cifras [Internet]. 2018. Disponible en: https://www.who.int/es/news-room/factsheets/detail/malnutrition

3. Ministerio de la Protección Social. Análisis de la situación de salud (ASIS) Colombia. Imprenta N. Bogotá D.C: Noviembre de 2016; 2016.

4. Adami F, Guedes de Vasconcelos FA. Childhood and adolescent obesity and adult mortality: a systematic review of cohort studies. Cad. Saude Pública. 2008;24(4). Disponible en:

http://www.scielo.br/scielo.php?script=sci_arttext\&pid=S0 102-311X2008001600008

5. Duran P, Caballero B de OM. The association between stunting and overweight in Latin American and Caribbean preschool children. Food Nutr Bull. DOI: http://dx.doi.org/10.1177/156482650602700403.

6. Secretaría de Salud Departamental. Boletín del estado nutricional de la población materno infantil en el Departamento del Huila, 2016. En 2016.

7. Barrera-Dussán N, Fierro-Parra E, Puentes-Fierro L, RamosCastañeda J. Prevalencia y determinantes sociales de malnutrición en menores de 5 años afiliados al Sistema de 
Selección de Beneficiarios para Programas Sociales (SISBEN) del área urbana del municipio de Palermo en Colombia, 2017. Univ. Salud. 2018;20(3):236-246. DOI: http://dx.doi.org/10.22267/rus.182003.126

8. Zelandia N. Datos de composición de alimentos y bases de datos de composición de alimentos. 1974.

9. Organización Mundial de la salud Bolivia. ¿Estamos creciendo bien? Los nuevos patrones de crecimiento de la OMS. 2007. Disponible en: http://www.ops.org.bo/textocompleto/naiepi-patronescrecimiento.pdf

10. Ministerio de la Protección Social. Resolución 2465 de 2016. 2016;1-47. Disponible

en: https://www.minsalud.gov.co/Normatividad_Nuevo/Resolu cion\%202465\%20de\%202016.pdf

11. Organización Mundial de la Salud. Curso de Capacitación sobre la evaluación del crecimiento del niño. Ginebra, OMS. 2008;

12. Ministerio de Salud. Resolución 8430 de 1993. República de Colombia. 1993; 1993:12. Disponible en: https://www.minsalud.gov.co/sites/rid/Lists/BibliotecaDig ital/RIDE/DE/DIJ/RESOLUCION-8430-DE-1993.PDF
13. Sguassero DY, Moyano LC, Aronna LA. Validación clínica de los nuevos estándares de crecimiento de la OMS: análisis de los resultados antropométricos en niños de 0 a 5 años de la ciudad de Rosario, Argentina. Arch Argent Pediatr 2008;106(3):198-204. Disponible en: https://pesquisa.bvsalud.org/portal/resource/pt/lil486952

14. Sepulveda CN, Ladino L. Comparación de la clasificación antropométrica de cien niños entre los 2-18 años, según los estándares de crecimiento de la OMS 2006-2007 y las tablas de NCHS/CDC 2000. 2011;13(1):1016. Disponible en: http://revgastrohnup.univalle.edu.co/a11v13n1/a11v12n1 art2.pdf

15. Restrepo AE, Diego J, Rueda G. Análisis de datos antropométricos de la población menor de 18 años de Medellín usando los estándares de la Organización Mundial de la Salud y su adaptación para Colombia propuesta por el Ministerio de la Protección Social. 2012;14:33-45. 